\title{
Article \\ Effect of Fibre Diameter, Prickle Factor and Coarse Fibre Bias on Yarn Surface Hairiness in South American Camelids (SAC) Fibre
}

\author{
Ruben Herberht Mamani-Cato ${ }^{1, *} \mathbb{E}$, Eduardo Narciso Frank ${ }^{2, *} \mathbb{C}$, Alejandro Prieto ${ }^{3}$, Maria Flavia Castillo ${ }^{3}$, \\ Nicoll Condori-Rojas ${ }^{1}$ and Michel Victor Hubert Hick ${ }^{2}$
}

check for

updates

Citation: Mamani-Cato, R.H.; Frank,

E.N.; Prieto, A.; Castillo, M.F.;

Condori-Rojas, N.; Hick, M.V.H.

Effect of Fibre Diameter, Prickle

Factor and Coarse Fibre Bias on Yarn

Surface Hairiness in South American

Camelids (SAC) Fibre. Fibers 2022, 10,

18. https://doi.org/10.3390/

fib10020018

Academic Editor: Alan K. T. Lau

Received: 14 December 2021

Accepted: 29 January 2022

Published: 10 February 2022

Publisher's Note: MDPI stays neutral with regard to jurisdictional claims in published maps and institutional affiliations.

Copyright: (c) 2022 by the authors. Licensee MDPI, Basel, Switzerland. This article is an open access article distributed under the terms and conditions of the Creative Commons Attribution (CC BY) license (https:// creativecommons.org/licenses/by/ $4.0 /)$.
1 Estación Experimental Agraria Illpa, Dirección de Desarrollo Tecnológico Agrario, Instituto Nacional de Innovación Agraria (INIA), Rinconada Salcedo S/N KM 22 Carretera Puno-Juliaca, Paucarcolla, Puno 21000, Peru; nicoll.c.rojas@gmail.com

2 IRNASUS-CONICET-UCC-CONICET, Catholic University of Cordoba, Cordoba 5014, Argentina; michelhick.agro@ucc.edu.ar

3 Facultad de Ciencias Agropecuarias, Catholic University of Cordoba, Cordoba 5014, Argentina; 9708967@ucc.edu.ar (A.P.); mflaviacastillo@gmail.com (M.F.C.)

* Correspondence: rmamani@inia.gob.pe (R.H.M.-C.); frank.agro@ucc.edu.ar (E.N.F.)

\begin{abstract}
It is well known that objectionable fibres emerge from the surface of the yarn due to the centrifugal force of the spinning device. Furthermore, the hair removal process is based on the same physical principles. However, the fibres that are $>30 \mu \mathrm{m}(\mathrm{PcF})$ are the fibres that appear in the hairiness of the yarn and are eliminated by dehairing. It has always been presumed that the PcF was linearly correlated with the diameter of the fibre (MFD) in llamas, but not so in alpaca fibres. Nevertheless, there is evidence that this relationship is curvilinear and behaves the same way in both species. The objectives of this study are to explore the relationship between MFD and PcF in both llamas and alpacas, to explore the existence of a breaking point (BP) in this curvilinear relationship, and to determine the frequency of fleeces that do not require dehairing because the $\mathrm{PCF}_{\mathrm{C}} \leq 3.2 \%$. In addition, the existence of a positive bias of coarse fibre content on the hairy surface (CFs) of the yarn to coarse fibre content within the yarn fibres (CFy) was determined, which may explain the effect of the dehairing on the prickle factor of SAC fibres. The relationship of PcF on MFD behaves the same way in alpacas and llamas. It conforms to a power distribution and presents a BP of $23 \mu \mathrm{m}$, with PcF being constant before the BP and increasing significantly after it. Most animals ( $\leq 91 \%$ of alpacas and $\leq 87 \%$ of llamas) are above the threshold $(\leq 3.2 \%)$, requiring dehairing to correct it. By means of a shaving technique on the surface of the fabric sample, it was established that the objectionable CFs content is $8.15 \%$ higher than the objectionable CFy content. In the evoked-coarse fibre in the dehaired samples, a CFs-CFy difference below $5.9 \%(p>0.05)$ is not significantly detected by panellists. The surface MFD is more than $2.7 \mu \mathrm{m}$ coarser than the yarn MFD.
\end{abstract}

Keywords: itching; hairy yarns; alpaca and llama fibre; dehairing

\section{Introduction}

The South American domestic camelids' (SAC) (alpaca and llama) fibres are valued for their softness. However, for garments made with fine yarns, the prickle factor $(\mathrm{PcF})$ of the fibres is too high and is therefore negatively perceived by the consumer [1].

It is well known that objectionable fibres emerge from the surface of the yarn due to the centrifugal force of the spinning head. Furthermore, the hair removal process (dehairing) is based on the same physical principles [1]. However, the fibres $>30 \mu \mathrm{m}(\mathrm{PcF})$ are the fibres that appear in the hairiness of the yarn and are eliminated by dehairing. It has always been presumed that the PcF was linearly correlated with the diameter of the fibre (MFD) in llama fibres, but not so in alpaca fibres. Nevertheless, there is evidence that this relationship is curvilinear and behaves the same way in both species. 
In wool, the threshold is $\approx 3 \%$ of fibres $>30 \mu \mathrm{m}$ [2], and in SAC fibres, this value is similar for knitted fabric [1]. If the relationship between MFD and PcF were linear and positive, the finer fleeces would have a lower PcF; however, this relationship obeys a power law function [3], which is associated with the bias of the fibre diameter distribution [4]. A correlation was found between $\mathrm{PcF}$ and $1 / \mathrm{Z}$, where $\mathrm{Z}$ is the standard Gaussian score; however, this linear relationship breaks down both for small and large values of PcF [3]. A positive linear correlation was obtained in wool fabrics between the log-transformed prickle score and the mean fibre diameter $(\mathrm{r}=0.85)$. This indicated that a wearer prickle score of 2.0 is equivalent to no detection of prickle, and a score of 2.5 is equivalent to a slight detection of prickle [5].

Fabric-evoked prickle has been shown to derive from the coarser fibres in the fibre diameter distribution (coarse edge) [6]. Experiments with acrylic samples have shown that the percentages of fibres greater than 30-32 $\mu \mathrm{m}$ correlate positively with prickle or itching [7], and this threshold is similar for wool [8]. When working with dehaired and non-dehaired llama fibres, a similar result was obtained. A threshold of $3.2 \%$ of coarse fibres was significantly associated with itch or prickle factor when non-trained subjects assessed comfort sensation [1].

The coarse edge of the diameter distribution of fibre ends, i.e., the percentage of fibre ends greater than $32 \mu \mathrm{m}$, is commonly used in assessing potential levels of prickle in wool samples [9]. Nevertheless, the effect appears to be more dependent on the protruding fibres of the fabric than those that make up the yarn as a whole. Thus, in woollen fabrics of different origins, the fibre diameter distribution of fabric surface hairs suggest that during spinning, there is a small bias of the coarse fibre ends to the surface [7]. Similar results were obtained with llama fibres under a fabric consumer test [1].

Preliminary measurements of the CFs and CFy suggest that during spinning, there is a bias in CFs, highlighting that the coarse fibres $(\%>30 \mu \mathrm{m})$ contained in CFs are twice that contained in CFy [8]. This has already been explained at length many years ago in working with cotton; this phenomenon was due to the centrifugal force of the spinning head, which rotates at very high speeds $(9000-12,000 \mathrm{rpm})[10,11]$. A similar difference between $\mathrm{CFs}$ and $\mathrm{CFy}$ has also been detected in llama fibre fabrics [1]. A strong negative correlation between flexural rigidity (bending rigidity) and the number of protruding fibre ends was obtained in yarns made with cotton fibres $(r=-0.66)$ [10].

The objectives of this work are to explore the functions that fit the relationship between diameter (MFD) and prickle factor (PcF) in both llama and alpaca fibres belonging to different biotypes, to explore the possible existence of a breaking point (BP) in this curvilinear relationship, and also to show the frequency of fleeces that do not require dehairing because their $\mathrm{PcF} \leq 3.2 \%$. In addition, a the possible existence of a positive bias of coarse fibres on the hairy surface (CFs) of the yarn to the whole yarn fibres (CFy) was determined, which may explain the favourable effect of dehairing on the prickle factor of llama and alpaca fibres from the Peruvian populations.

\section{Materials and Methods}

The analysed data of MFD and PcF of 1090 llamas and 1338 alpacas belonged to the experimental station of the Peruvian National Institute for Agrarian Innovation (INIA) located in the Santa Lucia District, Puno Department, Quimsachata, Peru. The fibre samples were initially processed in the INIA laboratory by OFDA to obtain MFD and PcF. The data were adjusted to non-linear models through a power function. Segmented linear regression was used to verify the fit to two lines separated by a threshold value or break point (BP) in MFD. The SegReg program was used to estimate a BP with a confidence interval and adjusted to model 3 segmented regressions according to the maximum value of the explanatory correlation ( $\mathrm{R}^{2}$ of the segmented regression) (CE) [12].

Eighteen pairs of knitted fabric $(10 \mathrm{~cm} \times 10 \mathrm{~cm})$ of similar densities and titles were obtained from the animal fibre laboratory (LAFTA) of the IRNASUS institute in Argentina. Each pair was made up of a fabric spun with dehaired fibres (D) and one spun with non- 
dehaired fibres (ND). The yarn properties and fabric properties are as follows: Linear density (Tex): D:25, ND:32; n: D: 45, ND: 71; hairiness: D:4.9, ND: 7.5; fabric tightness factor: D: 13 tex $^{1 / 2} / \mathrm{cm}, \mathrm{ND}: 15$; average fabric thickness: D: 0.82, ND: 0.97; and average fabric mass $\left(\mathrm{g} / \mathrm{m}^{2}\right)$ : D: 228, ND: 230. The process of dehairing, carding, spinning, and knitting was performed according to a textile standard laboratory process [13]. Fibre samples were graded according to the three different types of fleeces described in Argentina Llama [14].

Highly humidified fabric samples were frozen by a Peltier frozen microtome and shaved with a razor blade, and then the fabrics were disassembled to randomly separate the yarn pieces. Both the shaved fibres and the fibres contained in the yarns were analysed for their coarse fibre content ( $w / w$, near $1 \mathrm{mg}$ ) percentage (CFs and CFy), mean diameter (MFDs and MFDy), and frequency of fibres with continuous and lattice medulla (CMFs and CMFy; LMFs and LMFy) under a 500× microprojector [1].

Cross sections of the fibres were obtained with a freezing microscope and corrected with a man-made reference fibre (see Figure 1 ). The major axis and the minor axis were measured with a microprojector at $500 \times$. The bending rigidity $(\mathrm{Br})$ and buckling load $(\mathrm{Bl})$ were estimated with the following equations:

$$
\mathrm{Br}=E \pi a b^{3} / 4
$$

where: $a$ and $b$ are the major (long) half axis and the minor (short) half axis of the ellipse of the cross section of the fibre, respectively, and $E$ is the Young's modulus [15].

$$
\mathrm{Bl}=E \pi^{3} D^{4} / 31.4 l^{2}
$$

where: $E$ is the Young's modulus, $D$ is the diameter of the fibre, and $l$ is the free fibre length [16].

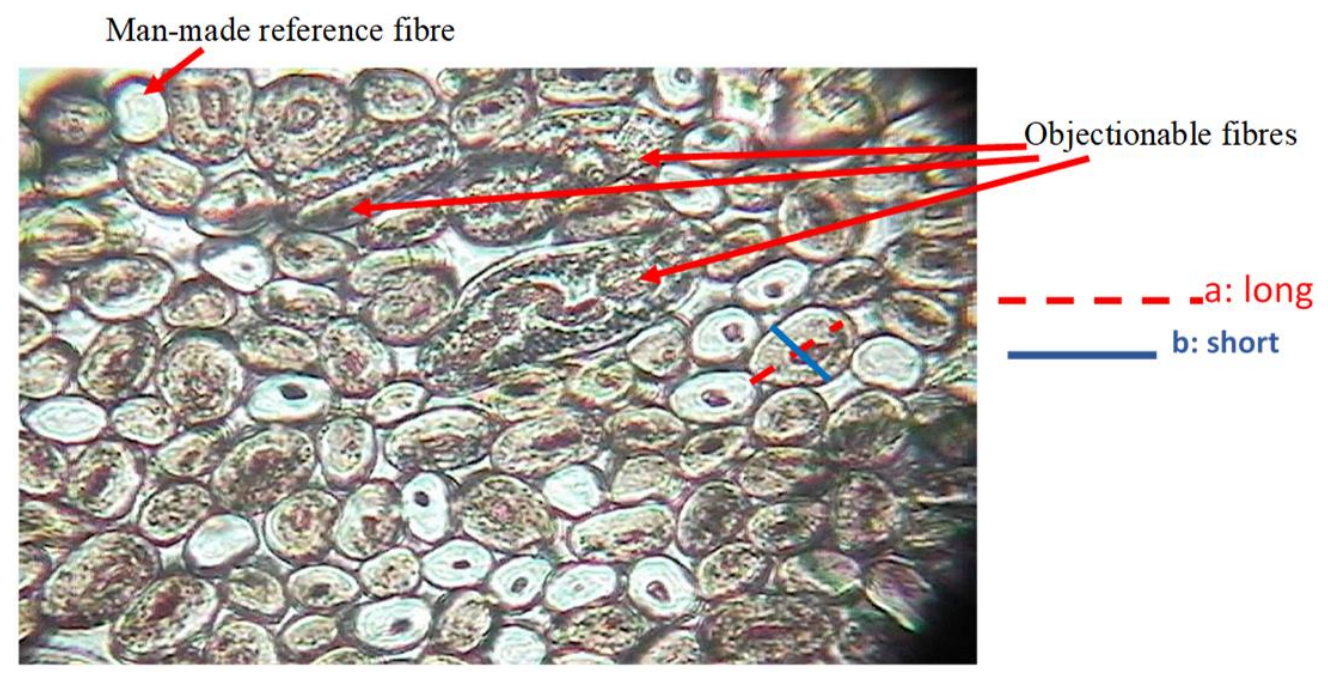

Figure 1. A microscopically pictured cross-section of the fibre sample with measurement indications as the $\mathrm{a}$ and $\mathrm{b}$ axes.

\section{Statistics}

The relationship between the mean fibre diameter of each fleece and the percentage of fibres $>30 \mu \mathrm{m}$ were fitted to a power law curve model according to Naylor (1996) [3]. The Nonlinear Mixed Models Module of the InfoStat program [17] was used.

Segmented linear regression was used to verify the fit to two lines separated by a threshold value or break point (PQ) in MFD. The SegReg program was used, which estimates a PQ with a confidence interval and adjusts to one of seven segmented regression models according to the maximum value of the explanatory correlation $\left(\mathrm{R}^{2}\right.$ of the segmented regression) (CE) [18]. 
The mean comparison of the variables of the dehaired fibre versus the non-dehaired fibre and versus within the yarn and on the surface of the yarn were compared by the ANOVA and DGC tests of the Generalized Mixed Linear Models module of the InfoStat program [17]. The same statistical procedure was used to compare the cross fibre diameter (MFDc), ellipticity (El), bending rigidity (Br), and buckling load (Bl) between fineness, type of wave, and type of medulla.

\section{Results and Discussion}

For the relationship of MFD over $\mathrm{PcF}_{\mathrm{f}}$, the function fit was somewhat better in the Huacaya Alpaca $\left(R^{2}: 0.91\right)$ and the Suri Alpaca $\left(R^{2}: 0.92\right)$ than in the $Q^{\prime}$ cara Llama $\left(R^{2}: 0.89\right)$ and the Chaqu Llama $\left(\mathrm{R}^{2}:\right.$ 0.84). A similar behaviour appears in wool [3] (Naylor, 1996) and in a work made of SAC fibres without making the distinction between biotypes within alpacas and within llamas [19].

The segmented regression was also similar in both species and biotypes. The break point (BP) in the Q'cara Llama = 23.2 $\mu \mathrm{m}$ and in the Chaqu Llama = $23.2 \mu \mathrm{m}$ (see Figure 2), and in alpacas, the BP of the Huacaya Alpaca $=23.0 \mu \mathrm{m}$ and the Suri Alpaca $=23.3 \mu \mathrm{m}$ (see Figures 2 and 3). The exploratory coefficient (EC) in the four biotypes was higher than the respective $\mathrm{R}^{2}$ without $\mathrm{BP}$, resulting in the line $<B P$ being non-significant and $>\mathrm{BP}$ being significant, indicating a relation to the model 3 function type of segmented regression (first a horizontal segment, then a sloping segment) and an increase of $\approx 1 \% / \mu \mathrm{m}$ and $\approx 5 \% / \mu \mathrm{m}$, respectively. A few of the fleeces, which do not need to be dehaired, reach the threshold of $\mathrm{PcF} \leq 3 \%$ (3.5\% and 3.8\% for alpacas and llamas, respectively). This figure is very similar to the $3.2 \%$ obtained with Argentine llama fibre fabrics [1].
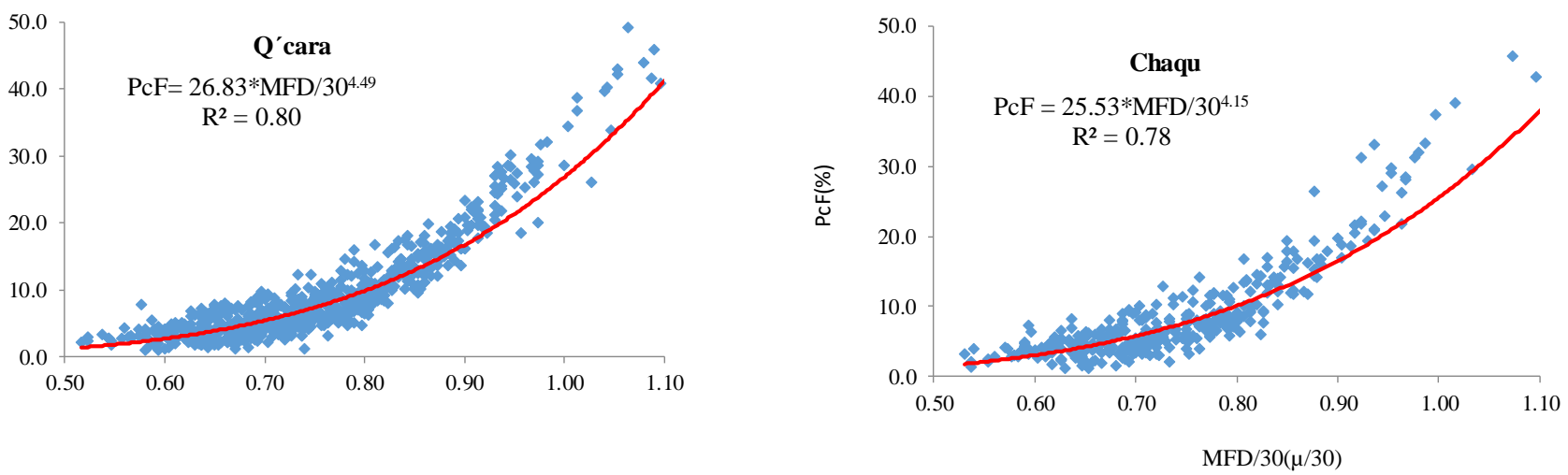

Figure 2. The percentage of fibres $>30 \mu \mathrm{m}$ on MFD/30 $\mu \mathrm{m}$ in two llama biotypes.
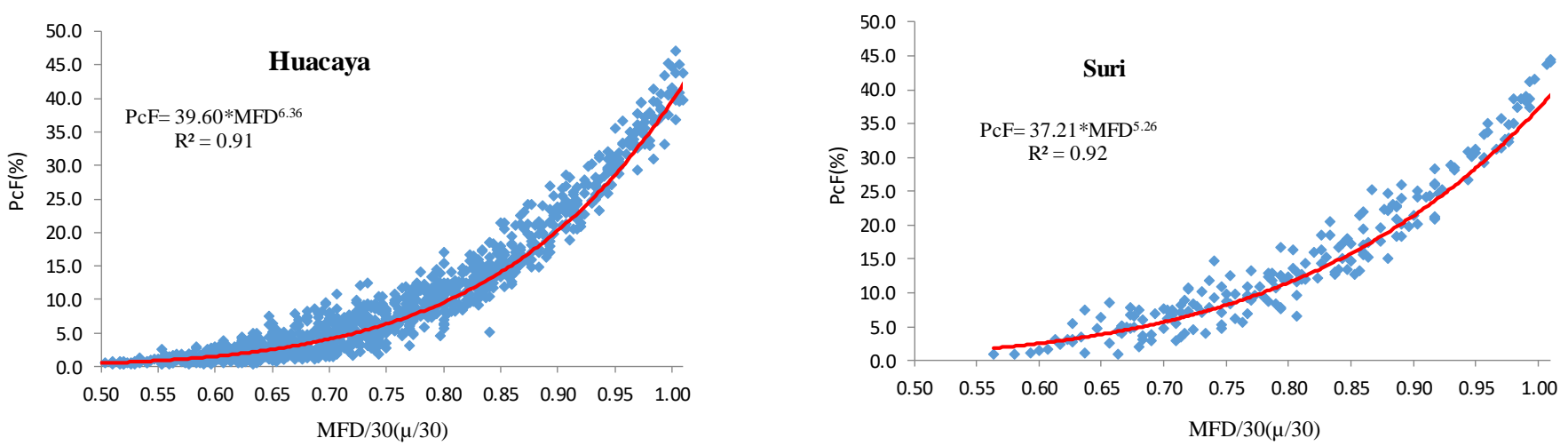

Figure 3. The percentage of fibres $>30 \mu \mathrm{m}$ on MFD/30 $\mu \mathrm{m}$ in two alpaca biotypes.

The relationship between MFD and PcF behaves the same way in alpacas and llamas (see Table 1). It conforms to a power law distribution and has a BP of $23 \pm 0.29 \mu \mathrm{m}$, and the $\mathrm{PcF}$ is constant before the BP and increases 5\% after it. Virtually all animals $(\leq 91 \%$ of 
alpacas and $\leq 87 \%$ of llamas) are above the threshold of prickle factor $(\leq 3.2 \%)$ and therefore would require dehairing (see Figures 2 and 3).

Table 1. The mean fibre diameter (MFD) and prickle factor $(\mathrm{PcF})$ averages below the breaking point $(<\mathrm{BP})$ and above the breaking point $(>\mathrm{BP})$ in llamas and alpaca fibres.

\begin{tabular}{|c|c|c|c|c|c|c|c|c|}
\hline Sp. & Biotype & & Overall & BP & $<\mathrm{BP}$ & $>\mathrm{BP}$ & Slope $<\mathrm{BP}_{\mathrm{MFD}}$ & Slope $>\mathbf{B P}_{\mathrm{MFD}}$ \\
\hline \multirow[t]{4}{*}{ Llama } & $Q^{\prime}$ cara & MFD & 23.69 & 23.20 & 20.40 & 26.20 & & \\
\hline & & $\mathrm{PcF}$ & 11.05 & 5.04 & 5.20 & 17.10 & 1.04 & 4.00 \\
\hline & Chaqu & MFD & 20.91 & 22.10 & 21.00 & 25.30 & & \\
\hline & & $\mathrm{PcF}$ & 8.81 & 5.54 & 4.70 & 18.00 & 1.10 & 3.90 \\
\hline \multirow[t]{4}{*}{ Alpaca } & Huacaya & MFD & 23.33 & 23.00 & 20.00 & 27.30 & & \\
\hline & & $\mathrm{PcF}$ & 14.15 & 3.89 & 3.90 & 26.60 & 1.04 & 5.50 \\
\hline & Suri & MFD & 25.87 & 23.42 & 21.00 & 28.30 & & \\
\hline & & $\mathrm{PcF}$ & 23.38 & 6.69 & 8.30 & 32.00 & 3.14 & 5.13 \\
\hline
\end{tabular}

The mean difference obtained $(8.2 \%)$ when conducting a paired sample t-test between CFs and CFy was highly significant $(p<0.0001)$, and the lower one-sided confidence interval was $5.94 \%(p<0.05)$. This difference is more than twice that obtained with woollen yarn [8]. This difference, taking into account the dehairing effect perception of the panellists, is analysed in Table 2 .

Table 2. The mean comparison of coarse fibre (CF) content (s: surface; y: yarn), fibre diameter (MFD) (s:y), and the difference between surface and yarn (Diff.S-Y), in relation to the interaction of dehairing (D; ND) per perception effects (0: non-sig.; 1: sig.).

\begin{tabular}{ccccccccccccc}
\hline Treat. & CFs & Sig & CFy & Sig & Diff.S-Y & Sig & MFDs & Sig & MFDy & Sig & Diff.S-Y & Sig \\
\hline D:1 & 5.7 & A & 4.7 & A & 1.0 & A & 24.5 & A & 23.1 & A & 1.5 & A \\
ND:1 & 15.7 & B & 9.7 & AB & 6.0 & B & 27.2 & AB & 24.8 & A & 2.5 & AB \\
D: 0 & 18.0 & BC & 9.1 & AB & 8.9 & B & 28.9 & B & 24.8 & A & 4.2 & B \\
ND:0 & 23.2 & C & 11.1 & B & 13.5 & B & 29.1 & B & 25.3 & A & 3.8 & B \\
\hline
\end{tabular}

The difference of about one point between the surface (CFs) and the yarn (CFy) in the dehaired samples, where the panellists ascertained significant difference from the non-dehaired samples, shows the corrective effect of this textile process [1] (see Figure 4). The difference between the types of fibres (visualised by medulla) is depicted in Table 2. Different types of fibres show major differences from whole fibre diameters (see Table 3).

\section{Dehaired Llama fibre fabric}

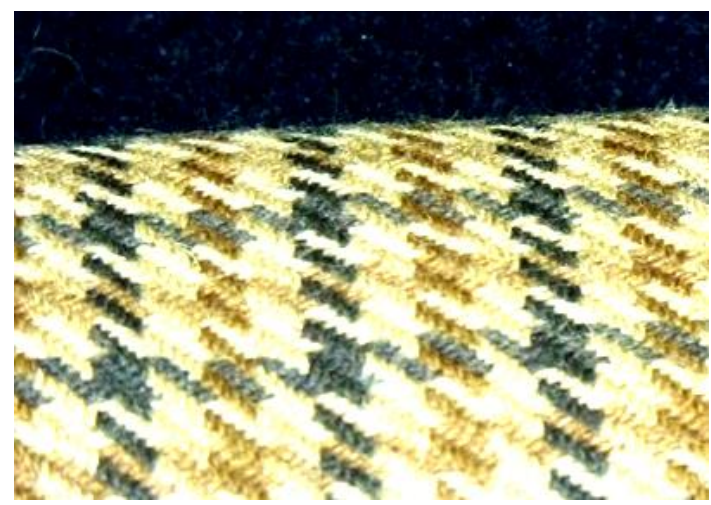

(a)

\section{Non-Dehaired Llama fibre fabric}

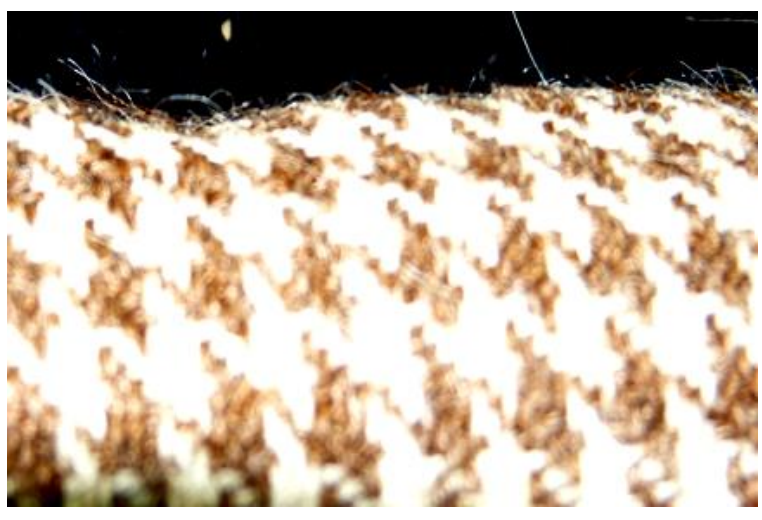

(b)

Figure 4. The two fabric samples used in the panellist test. (a) Dehaired llama fibre fabric; (b) Nondehaired llama fibre fabric. 
Table 3. The mean comparison of the continuous medulla (CMF) content (s: surface; $y$ : yarn), the lattice medulla (LMF) content (s;y), and the difference between the surface and the yarn (Diff.S-Y), in relation to the interaction of dehairing (D; ND) per perception effects (0: non-sig.; 1: sig.).

\begin{tabular}{ccccccccccccc}
\hline Treat. & CMFs & Sig & CMFy & Sig & Diff.S-Y & Sig & LMFs & Sig & LMFy & Sig & Diff.S-Y & Sig \\
\hline D:1 & 28.4 & A & 22.3 & A & 6.1 & A & 1.1 & A & 1.6 & A & -0.5 & A \\
ND:1 & 22.3 & A & 23.7 & A & -1.4 & A & 3.5 & B & 4.1 & B & -0.6 & A \\
D: 0 & 33.7 & A & 23.7 & A & 10.0 & B & 3.6 & B & 5.2 & B & -1.6 & A \\
ND:0 & 41.1 & A & 23.7 & A & 17.5 & B & 2.1 & A & 3.6 & B & -1.6 & A \\
\hline
\end{tabular}

Means with a common letter are not significantly different $(p>0.05)$

References: D:1: dehaired and where comfort factor had a significant effect on the panellist; ND:1 non-dehaired and where comfort factor had a significant effect on the panellist; D:0: dehaired and where comfort factor had a non-significant effect on the panellist; ND:1: non-dehaired and were comfort factor had a non-significant effect on the panellist.

It is clear that there is a positive bias of coarse fibres on the hairy surface of the yarn to the whole yarn, which may explain the favourable effect of dehairing on the prickle factor of llama and alpaca fibres (>5.9\%). Also, in dehaired fibres, a significant difference in prickle factor is perceived by the panellist when the difference between the hairy surface and the whole yarn is under this value.

A strong relationship exists between the amount of protruding fibres and the stiffness of the fibres. The stiffness is largely determined by the diameter of the fibre; therefore, the diameter of the fibres in the cross section was estimated to obtain ellipticity, the $\mathrm{Br}$ of the fibres in the longitudinal direction, and the $\mathrm{Bl}$ of a fibre that protrudes $2 \mathrm{~mm}$ above the fabric. The results are shown in Table 4 in relation to the degree of fineness, the type of wave, and the type of medulla of the fibre. The types of fibre, defined by fineness, type of wave, and medulla, are significantly different for $\mathrm{El}, \mathrm{Br}$, and $\mathrm{Bl}$; similar results were obtained with fibre diameter, curvature, number of scales, height of scale, and so on [1].

Table 4. The cross fibre diameter (MFDc), ellipticity (El), bending rigidity (Br), and buckling load (Bl) of fibres in relation to fineness, type of wave, and type of medulla.

\begin{tabular}{|c|c|c|c|c|c|c|c|c|}
\hline Fineness & MFDc & Sig & El & Sig & $\mathrm{Br}$ & Sig & B1 & Sig \\
\hline SF & 24.35 & $\mathrm{~A}$ & 1.19 & A & $5.86 \times 10^{9}$ & $\mathrm{~A}$ & 19.60 & A \\
\hline $\mathrm{F}$ & 32.55 & B & 1.28 & $\mathrm{~A}$ & $1.12 \times 10^{10}$ & A & 47.13 & A \\
\hline M & 44.09 & $\mathrm{C}$ & 1.43 & B & $3.36 \times 10^{10}$ & B & 841.38 & B \\
\hline$S$ & 52.06 & $\mathrm{D}$ & 1.60 & $\mathrm{C}$ & $5.63 \times 10^{10}$ & $\mathrm{C}$ & 1954.59 & $\mathrm{C}$ \\
\hline SS & 80.28 & $\mathrm{E}$ & 1.68 & $\mathrm{C}$ & $2.48 \times 10^{11}$ & $\mathrm{D}$ & 6548.33 & $\mathrm{D}$ \\
\hline \multicolumn{9}{|l|}{ Wave } \\
\hline 6 & 24.35 & A & 1.19 & $\mathrm{~A}$ & $5.86 \times 10^{9}$ & A & 19.60 & A \\
\hline 4 & 33.09 & B & 1.33 & B & $1.51 \times 10^{10}$ & $\mathrm{AB}$ & 446.33 & B \\
\hline 5 & 41.14 & $\mathrm{C}$ & 1.44 & $\mathrm{C}$ & $2.22 \times 10^{10}$ & $\mathrm{AB}$ & 500.90 & B \\
\hline 3 & 45.41 & $\mathrm{D}$ & 1.47 & C & $3.16 \times 10^{10}$ & B & 816.73 & $\mathrm{C}$ \\
\hline 2 & 54.62 & $\mathrm{E}$ & 1.49 & C & $5.54 \times 10^{10}$ & $\mathrm{C}$ & 1009.63 & $\mathrm{C}$ \\
\hline 1 & 79.31 & F & 1.88 & $\mathrm{D}$ & $2.54 \times 10^{11}$ & $\mathrm{D}$ & 4410.57 & $\mathrm{D}$ \\
\hline \multicolumn{9}{|l|}{ Medulla } \\
\hline $\mathrm{Nm}$ & 31.53 & A & 1.11 & A & $3.67 \times 10^{9}$ & A & 61.11 & A \\
\hline $\mathrm{F}$ & 32.46 & A & 1.17 & $\mathrm{~A}$ & $5.51 \times 10^{9}$ & A & 438.66 & B \\
\hline I & 36.99 & B & 1.28 & B & $1.21 \times 10^{10}$ & A & 411.35 & B \\
\hline $\mathrm{C}$ & 47.4 & $\mathrm{C}$ & 1.61 & $\mathrm{C}$ & $4.43 \times 10^{10}$ & B & 833.56 & $\mathrm{C}$ \\
\hline $\mathrm{L}$ & 59.71 & $\mathrm{D}$ & 1.80 & $\mathrm{D}$ & $1.02 \times 10^{11}$ & $\mathrm{C}$ & 3753.72 & $\mathrm{D}$ \\
\hline
\end{tabular}

Means with a common letter are not significantly different $(p>0.05)$.

References: Fineness: SF: super fine (<21 $\mu \mathrm{m})$; F: fine $(21.9-26 \mu \mathrm{m})$; M: medium $(26-30 \mu \mathrm{m})$; S: strong $(30-35 \mu \mathrm{m})$ SS: super strong $(>35 \mu \mathrm{m})$. Type of Wave: 6: close crimped; 5: regular crimped; 4: irregular crimped; 3: small crimped; 2: low crimped; 1 : virtually straight. Type of Medulla: Nm; non-medullated; F: fragmented medulla; I: interrupted medulla; C: continuous medulla; L: lattice medulla. 
Some important correlations were obtained between MFD and $\mathrm{Br}(0.84)$ and $\mathrm{Bl}(0.82)$, similar to cotton fibre work [9]. Interesting correlations were also obtained with the degree of fibre curvature $(\mathrm{Br}:-0.71$ and $\mathrm{Bl}$ : -0.36$)$. The ellipticity of the fibre also explains the bending rigidity $(\mathrm{r}=0.50)$.

\section{Conclusions}

The function that relates the diameter of the fibre and the prickle factor is a power law function and is similar between alpaca and llama fibres, and even between biotypes of both species.

In this curvilinear fit curve, there is a break point at the $23 \mu \mathrm{m} \mathrm{MFD,} \mathrm{which} \mathrm{determines}$ a constant increase of $\mathrm{PcF}(1 \% / \mu \mathrm{m})$ below that threshold and a 5\% increase in PcF for each $1-\mu \mathrm{m}$ increase in the diameter of the fibre.

A similar frequency between alpaca and llama fleeces is below the threshold of $<3.2 \%$; therefore, most of the fleeces of both species and both biotypes could be dehaired.

A very important conclusion of this work is that the fibre diameter of the yarn surface is more than $2.7 \mu \mathrm{m}$ coarser than inside the whole yarn when consumers detected a significant difference in the prickle factor, due to the presence of $6.1 \%$ more coarse fibres on the yarn surface than within the yarn. The greater bending rigidity that this fibre exhibits and the greater buckling load force of this type of fibre, both attributes are required to allow its possible extraction by dehairing, explain this higher percentage of objectionable fibres $(\%>30 \mu \mathrm{m})$ on the surface of the yarn, clearly demonstrated by the relation to the type of fibre detected by the type of medulla of this fibre.

Author Contributions: Conceptualization, E.N.F. and M.V.H.H.; methodology, E.N.F.; software, M.V.H.H.; validation, R.H.M.-C. and N.C.-R.; formal analysis, R.H.M.-C. and M.V.H.H.; investigation, A.P. and N.C.-R.; data curation, M.F.C.; writing-original draft preparation, E.N.F.; writing-review and editing, E.N.F.; visualization, M.V.H.H.; supervision, R.H.M.-C.; funding acquisition, R.H.M.-C. All authors have read and agreed to the published version of the manuscript.

Funding: This research was funded by Project 133 PI, CIP Quimsachata of INIA.

Institutional Review Board Statement: Not applicable.

Informed Consent Statement: Not applicable.

Data Availability Statement: Not applicable.

Acknowledgments: The data were collected as part of the Project 133_PI: "Formation of an elite genetic nucleus of breeding Huacaya Alpacas for the production of high-quality fibre at the CIPQuimsachata of INIA". It was funded by the National Agricultural Innovation Program of the National Institute of Agrarian Innovation (INIA), to which both the Peruvian and the Argentine authors are particularly grateful.

Conflicts of Interest: The authors declare no conflict of interest.

\section{References}

1. Frank, E.N.; Hick, M.V.H.; Castillo, M.F.; Prieto, A. Fibre-Based Components Determining Handle/Skin Comfort in Fabrics Made from Dehaired and Non Dehaired Llama Fibre. Int. J. Appl. Sci. Technol. 2014, 4, 51-66. Available online: https: //www.ijastnet.com/journal/index/583 (accessed on 19 December 2021).

2. Naylor, G.R.S.; Phillips, D.G.; Veitch, C.J.; Dolling, M.; Marland, D.J. Fabric-Evoked Prickle in Worsted Spun Single Jersey Fabrics Part I: The Role of Fiber End Diameter Characteristics. Text. Res. J. 1997, 67, 288-295. [CrossRef]

3. Naylor, G.R. The Coarse Fibre Component and Fibre End Diameter Characteristics of Australian Wool Tops. J. Text. Inst. 1996, 87, 265-273. [CrossRef]

4. Gilmour, A.; Atkins, K. Modelling the FFDA fibre diameter histogram of fleece wool as a mixture distribution. Aust. J. Agric. Res. 1992, 43, 1777-1788. [CrossRef]

5. McGregor, B.A.; Naebe, M.; Stanton, J.; Speijers, J.; Beilby, J.; Pieruzzini, S.; Tester, D. Relationship between wearer prickle response with fibre and garment properties and Wool ComfortMeter assessment. J. Text. Inst. 2013, 104, 618-627. [CrossRef]

6. Garnsworthy, R.K.; Gully, R.L.; Kenins, P.; Mayfield, R.J.; Westerman, R.A. Identification of the physical stimulus and the neural basis of fabric-evoked prickle. J. Neurophysiol. 1988, 59, 1083-1097. [CrossRef] [PubMed] 
7. Naylor, G.R.S. The role of coarse fibers on fabric prickle using blended acrylic fibers of different diameters. Wool Technol. Sheep Breed. 1992, 40, 14-18.

8. Naylor, G.R.S. The Relationship between the Fibre Diameter Distributions of Wool Top, Fibre Ends and Yarn Surface Fibres. Wool Technol. Sheep Breed. 1992, 40, 40-43.

9. Naylor, G.R.S. Improving the Skin Comfort Characteristics of Wool Fabrics. In Proceedings of the Textile Institute Natural Fibres in Australasia Conference, Dunedin, New Zealand, 15-17 April 2009.

10. Pillay, K.P.R. A Study of the Hairiness of Cotton Yarns. Part I: Effect of Fiber and Yarn Factors. Text. Res. J. 1964, 34, 663-674. [CrossRef]

11. Seshan, K.N. An investigation of the taper of cotton fibres. part iv: The differential behaviour of fibre ends during the processing of cotton. J. Text. Inst. 1975, 66, 109-115. [CrossRef]

12. Oosterban, R.J. Statistical Significance of Segmented Linear Regression with Break-Point Using Variance Analysis (ANOVA) and F-Tests. 2017. Available online: https:/ / www.waterlog.info (accessed on 20 January 2021).

13. Frank, E.; Hick, M.; Adot, O. Descriptive differential attributes of type of fleeces in llama fibre and its textile consequence. Part 2 : Consequences of the dehairing process. J. Text. Inst. 2011, 102, 41-49. [CrossRef]

14. Frank, E.N.; Brodtmann, L.I.; Hick, M.H.V. Multivariate Analysis for Fleece Types Classification in Argentine Llamas. J. Text. Sci. Fash. Technol. 2019, 3, 1-4. [CrossRef]

15. Wortmann, F.J.; Schwann-Jonczk, A. Investigating hair properties relevant fo hair 'Handle'. Part 1: Hair diameter, bending and frictional properties. Int. J. Cosmet. Sci. 2006, 28, 61-68. [CrossRef] [PubMed]

16. Veitch, C.J.; Naylor, G.R.S. The mechanisms of fiber buckling in relation to fabric-evoked prickle. Wool Technol. Sheep Breed. 1992, 40,31-34.

17. InfoStat, InfoStat versión 1.1; Grupo InfoStat, FCA, Universidad Nacional de Córdoba: Argentina, [Version 1.1 InfoStat. Group InfoStat, FCA. National Unviersity of Cordoba, Argentina]. 2002. Available online: https://www.infostat.com.ar/index.php? mod=page\&id=34(accessed on 12 December 2021).

18. Lerman, P.M. Fitting Segmented Regression Models. J. R. Stat. Soc. 1980, 29, 77-84. Available online: http://www.jstor.org/ stable/2346413 (accessed on 20 June 2019).

19. Frank, E.N.; Hick, M.H.V.; de Neyra, L.A.R. The Problem of Prickling on Fabrics of South American Camelids Fibers: Possible Approaches for Mechanical Solutions. Asian Res. J. Agric. 2017, 5, 1-9. [CrossRef] 